\title{
Giant malignant phyllodes tumor of the breast: A rare case report and literature review
}

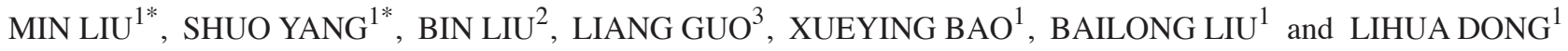 \\ Departments of ${ }^{1}$ Radiation Oncology, ${ }^{2}$ Hand Surgery and ${ }^{3}$ Pathology, \\ The First Hospital of Jilin University, Changchun, Jilin 130021, P.R. China
}

Received March 14, 2015; Accepted April 12, 2016

DOI: $10.3892 / \mathrm{ol} .2016 .4583$

\begin{abstract}
Malignant phyllodes tumor of the breast (MPTB) is rarely encountered in clinical practice. Preoperative diagnosis is challenging due to nonspecific radiological and histological features, and the prognostic factors and optimal treatment remain controversial. The current report describes the case of a middle-aged female with giant MPTB who underwent multidisciplinary intervention, including surgery, postoperative chemotherapy and radiotherapy. To date, the disease-free survival (DFS) of the patient has reached 18 months. Furthermore, a related literature review summarize the clinicopathological characteristics and treatment progress regarding MPTB is presented, along with an analysis of the indications for therapeutic strategy in the current case. In the future, multi-center clinical trials must be initiated to identify the criteria for diagnosis and optimal treatment consensus for MPTB. In conclusion, the present case highlights that multidisciplinary management may contribute to DFS following the treatment of giant MPTB.
\end{abstract}

\section{Introduction}

Malignant phyllodes tumor of the breast (MPTB) is a rare but distinct clinicopathological entity. MPTB typically occurs in middle-aged women (1), and has an average annual ageadjusted incidence of 2.1 per million females (2). As far as the etiology is concerned, Li-Fraumeni syndrome (germline TP53 mutation) has been reported to increase the risk of phyllodes tumors $(3,4)$. Surgery is the typical initial treatment option for MPTB and radiotherapy is recommended for individuals with

Correspondence to: Dr Bailong Liu or Dr Lihua Dong, Department of Radiation Oncology, The First Hospital of Jilin University, 71 Xinmin Street, Changchun, Jilin 130021, P.R. China

E-mail: bailong3385@163.com

E-mail: 1ijie200461@126.com

*Contributed equally

Abbreviations: BCS, breast conserving surgery; MPTB, malignant phyllodes tumor of the breast; PT, phyllodes tumor

Key words: malignant phyllodes tumor, breast, radiotherapy a high local recurrence risk. Chemotherapy is used to treat patients with a high systemic metastatic risk (3). Given its rarity, decisions regarding treatment options are based on small-scale retrospective clinical trials or case reports $(5,6,7)$. A rapidlygrowing breast mass is the most typical symptoms of MPBT and postoperative pathology is the most accurate method of diagnosis (3). Surgery is regarded as the primary method of management of MPBT. The clear margin achieved by surgery rather than the surgery type (breast conserving surgery or total mastectomy) determines the local recurrence rate (6). The five-year disease-free survival (DFS) is $60-90 \%$ (6). A previous study observed that $14.3 \%$ of patients died of metastatic MPTB 5 years following initial diagnosis (6). To date, the optimal intervention strategy has not been established. Herein, the case of a giant MPTB (the postoperative specimen measured $14.5 \times 10.5 \times 4.5 \mathrm{~cm}$ ), in which surgery, adjuvant chemotherapy and radiotherapy was performed, is presented. Written informed consent was obtained from the patient for the publication of her data in the present case report. In addition, the related literature was reviewed in order to expand our understanding of this unique breast malignancy.

\section{Case report}

A 43-year-old female was referred to The First Hospital of Jilin University in August, 2014 with a history of a painless lump in the right breast for 9 months. She had no other discomfort. Her past history was unremarkable, except allergies to starch and penicillin.

On physical examination, a large firm mass measuring about $11 \times 4.5 \mathrm{~cm}$ was palpated in the upper outer quadrant of right breast. A skin ulcer of $1.4 \mathrm{~cm}$ was present on the surface of the mass. In the bilateral supra- and subclavicular regions and axillas, there were no palpable enlarged lymph nodes.

Laboratory investigations revealed that the complete blood cell count and serum biochemical profile were normal. Ultrasound (Philips iU22; Philips Medical Systems, Inc., Bothell, WA, USA) of the breast demonstrated an $11.2 \times 4.54-\mathrm{cm}$, heterogeneous, hypoechoic mass in the upper outer quadrant of right breast with blood flow. Mammography (Selenia Dimensions mammography system; Hologic Corporation, Bedford, MA, USA) also revealed an $11.0 \times 4.5 \mathrm{~cm}$ irregular mass of high density. The BI-RADS stage (8) was determined to be $4 \mathrm{~B}$ due to the observations of an unclear border and irregular lesion 

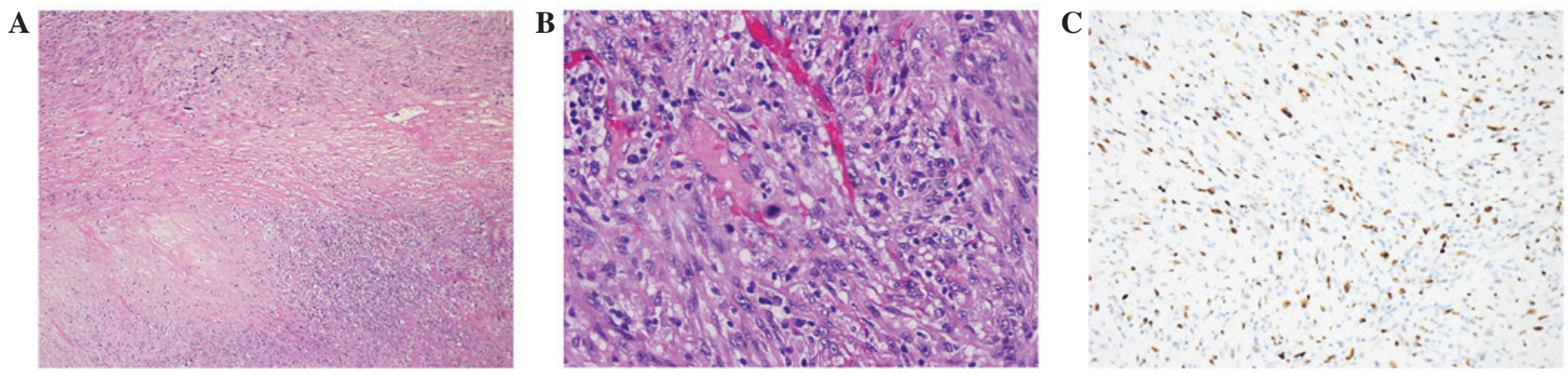

Figure 1. Pathological results of the postoperative specimen. (A) The tumor showed apparent necrosis (H\&E staining; magnification, $\mathrm{x} 100)$. (B) The neoplastic cells demonstrated a high mitotic rate (2-5 per high-power field), indicating highly aggressive behavior (H\&E staining; magnification, $\mathrm{x} 400)$. (C) Ki-67 was positive in the nuclei of $30 \%$ of the neoplastic cells (immunostaining; magnification, x200). H\&E, hematoxylin and eosin.

shape revealed by the ultrasound. The subsequent biopsy indicated mesenchymal malignancy, as hematoxylin and eosin staining revealed spindle neoplastic cells and immunohistochemistry demonstrated Ki-67 (30\%+) and AE1/AE3 (-). An abdominal ultrasound and bone scan yielded unremarkable results.

The patient's performance status was evaluated as 1 based on Eastern Cooperative Oncology Group criteria (9). The patient subsequently underwent a simple mastectomy plus sentinel lymph node biopsy. Biopsy and postoperative specimens were fixed in formalin, embedded in paraffin, sliced and stained by hematoxylin and eosin (10). Immunohistochemistry was performed using the dex-tran-polymer method (EnVision+; Dako, Glostrup, Denmark) (10) using the following monoclonal mouse anti-human antibodies against Ki-67 (dilution, 1:200; catalog no., RMA-0542), AE1/AE3 (dilution, 1:200; catalog no., MAB-0049), cluster of differentiation (CD)34 (dilution, 1:200; catalog no., MAB-0034), desmin (dilution, 1:200; catalog no., MAB-0055), leukocyte common antigen (LCA; dilution, 1:200; catalog no. MAB-0037), smooth muscle actin (SMA; dilution, 1:200; catalog no., MAB-003), estrogen receptor (ER; dilution, 1:200; catalog no., MAB-0062), S-100 (dilution, 1:200; catalog no., RAB-0150), CD68 (dilution, 1:200; catalog no., MAB-0041) and cytokeratin (dilution, 1:200; catalog no., MAB-0049). All the antibodies were purchased from Fuzhou Maixin Biotechnology Development Co., Ltd., Fuzhou, China. Goat anti-mouse IgG horseradish peroxidse-conjugated secondary antibodies (MaxVision $^{\text {TM }}$ kit; catalog no., KIT-5010/5020/5030; Fuzhou Maixin Biotechnology Development Co., Ltd.) were incubated with the samples according to the manufacturer's protocol. The 3,3'-diaminobenzidine chromogenic reagent kit was also purchased from Fuzhou Maixin Biotechnology Development Co., Ltd., and used according to the manufacturer's protocol. The postoperative pathology revealed a high-grade malignant phyllodes tumor with multifocal necrosis (Fig. 1A). The primary tumor measured $14.5 \times 10.5 \times 4.5 \mathrm{~cm}$, with involvement of the skin, tissue beneath the nipple and superficial fascia, and a high mitotic rate (2-5 per high power field; Fig. 1B). There was no evidence of lymphovascular or neural invasion. The final immunohistochemistry results demonstrated a Ki-67 index of 30\%+ (Fig. 1C); negative reactivity for cytokeratin, AE1/AE3, CD34, desmin, LCA, SMA and ER(-); and diffuse positive reactivity for S-100 and CD68. Two sentinel lymph nodes proved negative for metastasis.
Postoperatively, 4 cycles of chemotherapy were administered. The first 2 cycles were as follows: Pirarubicin, $80 \mathrm{mg}$ $\left(50 \mathrm{mg} / \mathrm{m}^{2}\right)$, day 1; cyclophosphamide, $800 \mathrm{mg}\left(500 \mathrm{mg} / \mathrm{m}^{2}\right)$, day 1; and liposomal paclitaxel, $270 \mathrm{mg}\left(175 \mathrm{mg} / \mathrm{m}^{2}\right)$, day 2 . The 3rd and 4th cycles consisted of the following: Pirarubicin, $70 \mathrm{mg}$, day 1; cyclophosphamide, $700 \mathrm{mg}$, day 1; liposomal paclitaxel, $270 \mathrm{mg}$, day 2. Prophylactic chest irradiation was subsequently performed, with a dose of $60 \mathrm{~Gy} / 30$ fractions. To date, the patient has a good quality of life and has demonstrated DFS for 18 months.

\section{Discussion}

Phyllodes tumors (PTs) are rather rare entities, accounting for only $1 \%$ of all breast tumors (1). In 2003, the World Health Organization classified PTs into three subtypes, designated benign, borderline and malignant, according to various clinicopathological characteristics, including the degree of stromal cell atypia and stromal overgrowth, tumor necrosis, the status of mitosis and the tumor margin. (11-13). An extensive literature review was performed in the Pubmed database (www.ncbi.nlm.nih.gov/pubmed) using the following key terms: 'Malignant phyllodes tumor and breast'. The search was performed on the titles of English language articles published between 1993 and 2015. The relevant literature was studied to gain a full-scale understanding of MPTB. In the United States, 500 new MPTB cases are diagnosed annually (14). A fast-growing breast mass is the most common manifestation. Giant MPTB can lead to the development of hypoglycemia, which results from increased insulin-like growth factor 2 levels produced by the tumor $(15,16)$. Metastasis from MPTB occurs in $6.2-25 \%$ of cases (17), most frequently metastasizing to the lung, bone and liver $(18,19)$.

Imaging procedures, such as ultrasound and mammography, are barely able to differentiate PT from fibroadenoma $(3,20)$. In a previous study, mammography diagnosed PT in only $32 \%$ of cases (20). In addition, fine needle aspiration cytology or even core biopsy are usually inadequate for accurate diagnosis of PT (20). According to the report by Salvadori et al (20), cytology was performed on 30 patients in their study, and was suggestive of PT in only 4 cases.

Surgery is the primary option for the treatment of MPTB. For a long time, the extension of resection, namely the choice between breast-conserving surgery (BCS) and mastectomy, 
has remained controversial. Wide resection with clear margins of $\geq 1 \mathrm{~cm}$ has been recommended $(21,22)$. However, research by Belkacémi et al (23) revealed that, for borderline and malignant PTs of the breast, total mastectomy was superior to BCS in order to guarantee adequate safe margins. Mituś et al (6) supported that a tumor-free margin of $\geq 1 \mathrm{~cm}$ is critical for favorable local control following mastectomy or BCS. MPTB has a propensity for rare metastasis to the axillary lymph nodes, described in $<10 \%$ of cases; therefore, axillary dissection is not routinely performed $(24,25)$.

Positive surgical margins and large primary tumor have been proved to be poor prognostic factors for local recurrence (1). For patients with MPTB measuring $>2 \mathrm{~cm}$ after lumpectomy, or tumors $>10 \mathrm{~cm}$ after mastectomy, adjuvant radiotherapy is strongly recommended to control the high local relapse rate of $\geq 15 \%$ (26). Barth $\mathrm{Jr}$ et al (14) held the view that, for all the borderline and malignant breast PTs after margin-negative BCS, radiotherapy should be initiated. In their multi-institutional study, despite a median negative margin of $0.35 \mathrm{~cm}$ after $\mathrm{BCS}$, the local relapse rate was unexpectedly high at $21 \%$. Postoperative radiotherapy was effective in reducing the local recurrence, which is considered to be a strong indicator of distant metastases $(23,26,27)$ and is associated with significantly increased risk of mortality (26). Furthermore, given the fact that the most common local relapses occurred in the site of initial resection by BCS, partial breast irradiation may be as effective as whole breast irradiation (14). In the current case, the giant primary tumor $(14.5 \times 10.5 \times 4.5 \mathrm{~cm})$ and close margin (involvement of superficial fascia) indicated a high risk of local recurrence, making timely radiotherapy important for local control. Radiotherapy is also an effective treatment for symptomatic metastases of MPTB (28).

To date, the role of adjuvant chemotherapy remains controversial; cisplatin and etoposide, and ifosfamide alone or in combination with doxorubicin have been proven to be effective $(29,30)$. For large tumors ( $>5 \mathrm{~cm}$ ) or recurrent MPTB, chemotherapy is beneficial (29). The epithelial element of PT partially expresses ER (58\%) or progesterone receptor (75\%); however, there is no evidence that endocrine therapy is beneficial for treating PTs (31).

In conclusion, MPTB is a rare entity with distinct clinicopathological features. There is no established consensus regarding the optimal type of surgery and indications for radiotherapy and chemotherapy regimens. In the current case of a giant MPTB, multidisciplinary interventions contributed to favorable outcomes. In the future, large-scale multi-institutional clinical trials should be launched to clarify the indication for radiotherapy for MPTB.

\section{Acknowledgements}

The present study was supported by Traditional Chinese Medicine Administration Bureau of Jilin Province (grant no. 2014-Q54).

\section{References}

1. Jang JH, Choi MY, Lee SK, Kim S, Kim J, Lee J, Jung SP, Choe JH, Kim JH, Kim JS, et al: Clinicopathologic risk factors for the local recurrence of phyllodes tumors of the breast. Ann Surg Oncol 19: 2612-2617, 2012.
2. Bernstein L, Deapen D and Ross RK: The descriptive epidemiology of malignant cystosarcoma phyllodes tumors of the breast. Cancer 71:3020-3024, 1993.

3. Gradishar WJ, Anderson BO, Balassanian R, Blair SL, Burstein HJ, Cyr A, Elias AD, Farrar WB, Forero A, Giordano SH, et al: Breast Cancer, Version 1.2016. J Natl Compr Canc Netw 13:1475-1485, 2015.

4. Birch JM, Alston RD, McNally RJ, Evans DG, Kelsey AM, Harris M, Eden OB and Varley JM: Relative frequency and morphology of cancers in carriers of germline TP53 mutations. Oncogene 20: 4621-4628, 2001

5. Karczmarek-Borowska B, Bukala A, Syrek-Kaplita K, Ksiazek M, Filipowska $\mathbf{J}$ and Gradalska-Lampart M: A rare case of breast malignant phyllodes tumor with metastases to the kidney: Case report. Medicine (Baltimore) 94: e1312, 2015.

6. Mituś J, Reinfuss M, Mituś JW, Jakubowicz J, Blecharz P, Wysocki WM and Skotnicki P: Malignant phyllodes tumor of the breast: Treatment and prognosis. Breast J 20: 639-644, 2014.

7. Reinfuss M, Mituś J, Smolak K and Stelmach A: Malignant phyllodes tumours of the breast. A clinical and pathological analysis of 55 cases. Eur J Cancer 29A:1252-1256, 1993.

8. Sedgwick EL, Ebuoma L, Hamame A, Phalak K, Ruiz-Flores L, Ortiz-Perez T and Sepulveda KA: BI-RADS update for breast cancer caregivers. Breast Cancer Res Treat 150:243-254, 2015.

9. Oken MM,Creech RH, Tormey DC, Horton J, Davis TE, McFadden ET and Carbone PP.: Toxicity and response criteria of the Eastern Cooperative Oncology Group. Am J Clin Oncol 5: 649-655,1982.

10. Ma XB, Zheng Y, Yuan HP, Jiang J and Wang YP: CD43 expression in diffuse large B-cell lymphoma, not otherwise specified: CD43 is a marker of adverse prognosis. Hum Pathol 46: 593-599, 2015.

11. Lakhani SR, Ellis IO, Schnitt SJ, Tan PH and van de Vijver MJ (eds): WHO classification of tumours of the breast. In: IARC WHO Classification of Tumours. Vol 4. 4th edition. IARC Press, Lyon, 2012.

12. Wu DI, Zhang H, Guo L, Yan XU and Fan Z: Invasive ductal carcinoma within borderline phyllodes tumor with lymph node metastases: A case report and review of the literature. Oncol Lett 11: 2502-2506, 2016.

13. Bellocq JP and Magro G: Fibroepithelial tumours (Chapter 1 Tumours of the Breast). In: Pathology and Genetics - Tumours of the Breast and Female Genital Organs (World Health Organization Classification of Tumours). Tavassoli FA and Devilee P (eds). 5th edition. IARC Press, Lyon, France, pp99-103, 2003.

14. Barth RJ Jr, Wells WA, Mitchell SE and Cole BF: A prospective, multi-institutional study of adjuvant radiotherapy after resection of malignant phyllodes tumors. Ann Surg Oncol 16: 2288-2294, 2009.

15. Aguiar Bujanda D, Rivero Vera JC, Cabrera Suárez MA, Aguiar Morales J, Christol R, Bohn Sarmiento U, Domínguez Cabrera C and Le Bouc Y: Hypoglycemic coma secondary to big insulin-like growth factor II secretion by a giant phyllodes tumor of the breast. Breast J 13: 189-191, 2007.

16. Kataoka T, Haruta R, Goto T, Sugino K, Asahara T, Dohi K, Kaneco M, Arihiro K and Nomura S: Malignant phyllodes tumor of the breast with hypoglycemia: Report of a case. Jpn J Clin Oncol 28: 276-280, 1998.

17. Rowe JJ and Prayson RA: Metastatic malignant phyllodes tumor involving the cerebellum. J Clin Neurosci 22: 226-227, 2015.

18. Hlavin ML, Kaminski HJ, Cohen M, Abdul-Karim FW and Ganz E: Central nervous system complications of cystosarcoma phyllodes. Cancer 72: 126-130, 1993.

19. Al-Zoubaidi M, Qiu S, Bonnen M, Bonnen M, Joyner M, Roehl K, Silva C and Chao C: Malignant phyllodes tumor of the breast: A case report. Open Breast Cancer J 3: 45-48, 2011.

20. Salvadori B, Cusumano F, Del Bo R, Delledonne V, Grassi M, Rovini D, Saccozzi R, Andreola S and Clemente C: Surgical treatment of phyllodes tumors of the breast. Cancer 63: 2532-2536, 1989.

21. Barth RJ Jr: Histologic features predict local recurrence after breast conserving therapy of phyllodes tumors. Breast Cancer Res Treat 57: 291-295, 1999.

22. Khan SA and Badve S: Phyllodes tumors of the breast. Curr Treat Options Oncol 2: 139-147, 2001.

23. Belkacémi Y, Bousquet G, Marsiglia H, Ray-Coquard I, Magné N, Malard Y, Lacroix M, Gutierrez C, Senkus E, Christie D, et al: Phyllodes tumor of the breast. Int J Radiat Oncol Biol Phys 70: 492-500, 2008.

24. Khosravi-Shahi P: Management of non metastatic phyllodes tumors of the breast: Review of the literature. Surg Oncol 20: e143-e148, 2011.

25. Kraemer B, Hoffmann J, Roehm C, Gall C, Wallwiener D and Krainick-Strobel U: Cystosarcoma phyllodes of the breast: A rare diagnosis: Case studies and review of literature. Arch Gynecol Obstet 276: 649-653, 2007. 
26. Pezner RD, Schultheiss TE and Paz IB: Malignant phyllodes tumor of the breast: Local control rates with surgery alone. Int J Radiat Oncol Biol Phys 71: 710-713, 2008.

27. Kapiris I, Nasir N, A'Hern R, Healy V and Gui GP: Outcome and predictive factors of local recurrence and distant metastases following primary surgical treatment of high-grade malignant phyllodes tumors of the breast. Eur J Surg Oncol 27: 723-730, 2001.

28. Burton GV, Hart LL, Leight GS Jr, Iglehart JD, McCarty KS Jr and Cox EB: Cystosarcoma phyllodes: Effective therapy with cisplatin and etoposide chemotherapy. Cancer 63: 2088-2092, 1989.

29. Pacioles T, Seth R, Orellana C, John I, Panuganty V and Dhaliwal R: Malignant phyllodes tumor of the breast presenting with hypoglycemia: A case report and literature review. Cancer Manag Res 6: 467-473, 2014.
30. Hawkins RE, Schofield JB, Wiltshaw E, Fisher C and McKinna JA: Ifosfamide is an active drug for chemotherapy of metastatic cystosarcoma phyllodes. Cancer 69: 2271-2275, 1992.

31. Tse GM, Lee CS, Kung FY, Scolyer RA, Law BK, Lau TS and Putti TC: Hormonal receptors expression in epithelial cells of mammary phyllodes tumors correlates with pathologic grade of the tumor: A multicenter study of 143 cases. Am J Clin Pathol 118: $522-526,2002$. 